7

\title{
Stem cell mechanosensation on gelatin methacryloyl (GeIMA) stiffness gradient hydrogels
}

Claire Kim ${ }^{a}$, Jennifer L Young ${ }^{b, c}$, Andrew W Holle ${ }^{b, c}, K^{\prime}$ wanghee Jeong ${ }^{d}$, Luke G Major ${ }^{a}$, Ji Hoon Jeong ${ }^{\mathrm{e}}$, Zachary M Aman ${ }^{\mathrm{d}}$, Dong-Wook Han ${ }^{\mathrm{f}}$, Yongsung Hwang ${ }^{\mathrm{e}}$, Joachim P Spatz $^{b, c}$, Yu Suk Choi ${ }^{a, *}$

a School of Human Sciences, The University of Western Australia, 35 Stirling Highway, Crawley, Western Australia, 6009, Australia

b Department of Cellular Biophysics, Max Planck Institute for Medical Research, 69120, Heidelberg, Germany

c Department of Biophysical Chemistry, University of Heidelberg, D-69117, Heidelberg, Germany

d Fluid Science and Resources, Department of Chemical Engineering, School of Engineering, University of Western Australia, Perth, WA 6009, Australia

e Soonchunhyang Institute of Medi-bio Science, Soonchunhyang University, Cheonansi, Chungcheongnam-do 31151, Korea

$f$ Department of CognoMechatronics Engineering, College of Nanoscience \& Nanotechnology, Pusan National University, Busan 46241, Korea

${ }^{*}$ Corresponding author: Yu Suk Choi

School of Human Science

The University of Western Australia 35 Stirling Highway Crawley, Western Australia, 6009, Australia Tel: +61864887513

Email: yusuk.choi@uwa.edu.au 
1 Abstract:

2 Stiffness gradient hydrogels are a useful platform for studying mechanical interactions between cells and their surrounding environments. Here, we developed linear stiffness gradient hydrogels by controlling the polymerization of gelatin methacryloyl (GelMA) via differential UV penetration with a gradient photomask. Based on previous observations, a stiffness gradient GelMA hydrogel was created ranging from $\sim 4$ to 13 $\mathrm{kPa}$ over $15 \mathrm{~mm}(0.68 \mathrm{kPa} / \mathrm{mm})$, covering the range of physiological tissue stiffness

8 from fat to muscle, thereby allowing us to study stem cell mechanosensation and differentiation. Adipose-derived stem cells on these gradient hydrogels showed no durotaxis, which allowed for the screening of mechanomarker expression without confounding directed migration effects. In terms of morphological markers, the cell aspect ratio showed a clear positive correlation to the underlying substrate stiffness, while no significant correlation was found in cell size, nuclear size, or nuclear aspect ratio. Conversely, expression of mechanomarkers (i.e. Lamin A, YAP, and MRTFa) all showed a highly significant correlation to stiffness, which could be disrupted via inhibition of non-muscle myosin or Rho/ROCK signalling. Furthermore, we showed that cells plated on stiffer regions became stiffer themselves, and that stem cells showed stiffness-dependent differentiation to fat or muscle as has been previously reported in the literature.

Keywords: Mechanosensitive; Stiffness; Gradient; stem cell; differentiation 


\section{1. Introduction}

2 The microenvironment surrounding stem cells is known to impose a wide variety of chemical and mechanical cues that can influence stem cell differentiation and behaviour. [1] Stem cells must integrate these cues in order to enact changes in their function and to commit to specific cell lineages. Such dictation of stem cell behaviour via cocktails of chemical cues has been appreciated for decades, while mechanical influences and the notion of mechanosensation are more recent discoveries shifting the paradigm of stem cell biology.[1, 2]

In a process known as mechanotransduction, mechanical cues originating from the extracellular matrix (ECM) are converted into chemical signals via mechanosensitive pathways. Thus, recent studies have focused their attention on matrix stiffness, an intrinsic property of the ECM, and have shown that it is a key parameter in dictating many cellular functions, e.g. morphology, proliferation, differentiation, and migration.[1, 3] In the physiological environment, there exists a wide variation of stiffness across tissue types. When measured tissue stiffnesses are emulated as hydrogels in vitro (e.g. neurogenic tissue at $1 \mathrm{kPa}$, adipogenic at $3 \mathrm{kPa}$, myogenic at $12 \mathrm{kPa}$, and osteogenic at $30 \mathrm{kPa}$ ), adipose-derived or bone marrow-derived stem cells plated on these substrates have shown to be able to differentiate down specific cell lineages corresponding to the substrate stiffness of the tissue type on which they were plated. [1-4]

However, tissues are not always uniform; there also exist physiological gradients of stiffness at interfaces where contrasting environments are adjacent to one another, for example at neuromuscular junctions, muscle-tendon boundaries, and in fibrosis. [5] Pathological boundaries such as infarcted fibrous heart tissue adjoining healthy myocardium and a tumour within healthy tissue exist, thereby creating gradients of stiffness to which cells within the same tissue can sense and migrate along. [6] As cells are highly sensitive to tissue stiffness, the need for platforms capable of replicating such physiological stiffness gradients in vitro are of great value. To date, there have been numerous approaches for creating stiffness gradients in the lab. [7, 8] 
1 The most common polymers utilised in existing gradient systems, e.g. polyacrylamide,

2 lack native ECM compositions and have limited three-dimensional relevance due to 3 their cytotoxicity when encapsulating cells. [9] While many other promising systems that have three-dimensional relevance have been developed, most systems focusing on stem cell mechanobiology are fabricated as a discrete substrate stiffness.[10, 11] Furthermore, other current relevant gradient systems are lacking the dimensionality to avoid durotactic events e.g. steep gradients using polyacrylamide hydrogels. [8, 9, 1214] Thus, the ever growing field of mechanobiology requires reproducible stiffness gradient systems, with three-dimensional relevance, whereby the relationship between matrix stiffness and cell behaviour can be screened across multiple values and ranges.

Gelatin methacryloyl (GeIMA) is a commonly used biomaterial for stem cell differentiation and tissue engineering in the broad regenerative medicine field. [15] GelMA is a very versatile material due to the fact that its physical characteristics can be easily tuned to the user's requirements and its chemical composition of bioactive motifs permits direct cell-ECM interactions. [15] Additionally, cell encapsulation is possible due to its low cytotoxicity and the material itself is readily accessible and costeffective. [15] GelMA presents RGD ligands to cells bound on or within its polymer network. [15] These ligands preferentially engage integrin $\alpha 5 \beta 1$ and/or integrin $\alpha \mathrm{V} \beta 3$, allowing for cell attachment and the transmission of actomyosin-generated traction force to the bulk of the substrate. [3]

GelMA solutions can be polymerised to produce hydrogels via UV exposure after combining the pre-polymer GelMA with a photoinitiator such as Irgacure. The degree of polymerisation, which determines matrix stiffness, can be manipulated by controlling UV exposure time, UV energy level, GeIMA concentration, the methacrylation level of gelatin structures, and photoinitiator concentration. [15] GelMA stiffness can also be patterned through the use of photomasks that limit the penetration of UV light to initiate different degrees of polymerisation. This technique can be customised by the user with different ranges of photomask transparency. In this paper, we utilize a gradient transparency in the photomask in order to provide an avenue for incorporating a stiffness gradient into the hydrogel. 
2 By incorporating a stiffness gradient into a GelMA hydrogel, we can provide a platform suitable for characterising mechanosensitive protein responses to stiffness in a continuum, as well as develop the technology required for future translational studies and three-dimensional studies. While previous approaches have been developed to create stiffness gradients using GeIMA, e.g. a sliding UV photomask [16, 17] or sequential gelation with a mixture of other polymers, [18] none have focused their attention on stem cell mechanobiology or utilized the adipo-to-myo stiffness regime created using our method. In order to investigate stem cell mechanosensation on our stiffness gradient hydrogels, we analyzed cell migration, morphology, differentiation and characteristic mechanosensitive protein expression, i.e. Lamin A,YAP, and MRTFa.

\section{Materials and methods}

\subsection{GelMA synthesis}

Gelatin methacryloyl (GelMA) was synthesized by methacrylation of gelatin as described previously [19]. Briefly, 10 grams of gelatin powder was dissolved in 100 $\mathrm{mL}$ of phosphate buffered saline (PBS; Gibco) in a $500 \mathrm{~mL}$ round bottom flask and the mixture was heated at $60^{\circ} \mathrm{C}$ in an oil bath. Once gelatin was completely dissolved, 8 $\mathrm{mL}$ of methacrylic anhydride was added dropwise to the solution under vigorous stirring. After keeping the reaction mixture at $60^{\circ} \mathrm{C}$ for another two hours, $100 \mathrm{~mL}$ of pre-warmed PBS at $60^{\circ} \mathrm{C}$ was added to the mixture and kept at $60^{\circ} \mathrm{C}$ for another 30 minutes. The resulting GelMA solution was dialyzed using a dialysis tube (Spectrum Laboratories, \#132676) in deionized water at $40^{\circ} \mathrm{C}$ for seven days. This GelMA solution was lyophilized and stored at $-20^{\circ} \mathrm{C}$ until further use.

\subsection{Photomask fabrication}

Photomasks were used to regulate light exposure and generate stiffness gradient hydrogels. Using Seashore (free image editing software), $12 \times 12 \mathrm{~mm}^{2}$ photomasks with a $10-100 \%$ or $20-100 \%$ transparency gradient were designed. Repeats of these designs were then arranged in GIMP (free image editing software) and laser printed at 600 dpi onto transparent film (Fineline Print \& Copy, East Perth). 


\section{$1 \quad$ 2.3. Stiffness gradient hydrogel fabrication}

2 Freeze-dried GelMA was processed through a desiccator vacuum for ten minutes then

3 dissolved in $1 \mathrm{x}$ phosphate buffered saline (PBS; Gibco) to achieve a working concentration of $10 \%(\mathrm{w} / \mathrm{v})$. To dissolve and reduce viscosity, solution was then placed in a $37^{\circ} \mathrm{C}$ water bath for twenty minutes. Light-sensitive photoinitiator, Irgacure (2959; Sigma) prepared at $10 \%$ in ethanol, was then combined 1:100 with GelMA aliquoted into $1.5 \mathrm{~mL}$ microcentrifuge tubes wrapped in aluminum foil and stored at $4^{\circ} \mathrm{C}$ overnight. Glass microscope slides were treated with dichlorodimethylsilane (DCDMS; Sigma) and $15 \times 15 \mathrm{~mm}$ glass coverslips were cleaned by UV/Ozone ProCleaner ${ }^{\mathrm{TM}}$ (BioForce Nanosciences) for one minute per side, immersed in ethanol with $0.3 \%$ acetic acid and 0.005\% 3-(trimethoxysilyl)propylmethacrylate (3-TMPM; Sigma) for five minutes, then washed with ethanol and air-dried. The UV transilluminator (Chromato-Vue, UVP) using $15 \mathrm{~W}$ bulbs $(365 \mathrm{~nm}$ ) was pre-heated for ten minutes then a photomask of $20-100 \%$ transparency, covered by a DCDMS-treated slide, was placed on top. Onto the glass surface $50 \mu \mathrm{L}$ of the $10 \%$ GelMA and Irgacure solution was pipetted then sandwiched with a 3-TMPM-treated coverslip aligning with the photomask below. UV photopolymerization occurred for a duration of 1 minute and 30 seconds, then polymerized hydrogels were moved into sterile 12-well plates with PBS.

\subsection{Mechanical characterization using atomic force microscopy}

The stiffness gradient across the $15 \mathrm{~mm}$ hydrogel was verified via an MFP-3D Origin atomic force microscope (AFM; Asylum Research). Indents for compression measurements were applied by $200 \mu \mathrm{m}$ chromium/gold-coated, silicone-nitride triangular shaped cantilever tips with $17-\mathrm{kHz}$ resonant frequencies, and $0.08 \mathrm{~N} / \mathrm{m}$ force constants (Nano World model PNP-TR-50). Triplicate indents were taken in $1 \mathrm{~mm}$ intervals, approaching at $2 \mu \mathrm{m} / \mathrm{s}$ until a 2-nN trigger force was registered, and then retracting at $10 \mu \mathrm{m} / \mathrm{s}$. Contact-generated force graphs were analysed (IgorPro) to plot the linear stiffness gradient in kilopascals along distance using GraphPad Prism. [9] Cell stiffness measurement was done using the same method.

\subsection{Raman spectroscopy}

While the methods above are sufficient for determining the stiffness gradient present in the GeIMA sample, they do not determine the contribution of particular chemical 
1 bonds. Raman spectroscopy provides chemical information based on vibrational modes of molecular bonds, with each bond manifesting as a unique Raman band, enabling spatial characterization of the identified species [20]. In situ Raman spectroscopy delivers both non-invasive and semi-quantitative analyses [21]. In this work, two-dimensional line mapping was used to characterize the spatial profiles of amide groups in the GeIMA hydrogel. A square GelMA hydrogel $(11 \mathrm{~mm} \times 11 \mathrm{~mm} \times$ $0.5 \mathrm{~mm}$ ) with a vertical gradient of stiffness was prepared on a coverslip and placed on a temperature-controlled cell $\left(1^{\circ} \mathrm{C}\right)$. The cell was mounted on a 3 -axis translation stage in a Renishaw inVia confocal Raman microscope with a $532 \mathrm{~nm}$ laser excitation source. All measurements were conducted at a focal point $50 \mu \mathrm{m}$ above the coverslip surface, thus inside the hydrogel. Data acquisition and analysis were performed with WiRE 4.0 software (Renishaw). Two amide groups [22] were identified, and each curve was integrated to quantify the relative concentration profile of amide groups in the sample. First, a series of horizontal scans was conducted through four different axes. Each scan was followed by one in the opposite direction to estimate the relative concentration profile of the amide group measurements. Afterward, three vertical scans were conducted along the same axes as the horizontal scans to compare the known stiffness gradient with the population of amide groups in the hydrogel.

\subsection{Scanning electron cryomicroscopy (Cryo-SEM)}

Cryo-SEM was used to examine the native pore size across the cross-section of the hydrogel. For cross-section evaluation, GeIMA hydrogels were removed from their glass coverslip and mounted in a custom holder at a $90^{\circ}$ angle, flash frozen in liquid nitrogen, and freeze fractured with the Freeze Fracture System Leica EM BAF060 fitted with a VCT 100 BAS Rev 04 Controller (Leica Microsystems). The samples were then sublimated first from $-150{ }^{\circ} \mathrm{C}$ to $-120^{\circ} \mathrm{C}$ at a rate of $5^{\circ} \mathrm{C} / \mathrm{min}$, then from $-120{ }^{\circ} \mathrm{C}$ to $-90{ }^{\circ} \mathrm{C}$ at a rate of $2{ }^{\circ} \mathrm{C} / \mathrm{min}$, and finally held at $-90{ }^{\circ} \mathrm{C}$ for 1 hour. Samples were sputter-coated with $2 \mathrm{~nm}$ platinum before being transferred to the SEM (Zeiss Ultra 55 fitted with a Gemini Electron Beam and Baltec LE 02567 mn/140 Controller; Carl Zeiss NTS $\mathrm{GmbH}$ ) where they were evaluated at $5 \mathrm{kV}$ with a working distance of $\sim 6 \mathrm{~mm}$ using the secondary electron detector. Images were captured at multiple locations along the length of the hydrogel and recorded with $\mathrm{x}$ and $\mathrm{y}$ coordinates to correlate with the known direction of the stiffness gradient. [9] 


\section{$1 \quad$ 2.7. Stem cell culture and seeding}

2 Human adipose-derived stem cells (ASC-F-SL, ZenBio) from 5 different female donors

3 (age: 29-65; location: abdomen, flanks, thigh) were cultured in growth media (15\%

4 fetal bovine serum and 1\% 1x antibiotic- antimycotic in Dulbecco's Modified Eagle

5 Medium; Gibco) and used at passage five. ASCs were seeded onto stiffness gradient

6 hydrogels at 20,000 cells/well (12 well plate) and incubated in growth media for a

7 period of 3 days for mechanosensitive markers and 5 days for differentiation markers.

8 A separate set of seeded stiffness gradient hydrogels were treated with non-muscle

9 myosin II inhibitor, $50 \mu \mathrm{M}$ Blebbistatin (Sigma), and ROCK inhibitor, $10 \mu \mathrm{M}$ Y-27632

10 (Sigma) for 24 hours. ASCs were cultured for another 2 days prior to

11 immunocytochemistry.

\subsection{Cell migration}

A Nikon Ti-E inverted microscope, Nikon Fi2 camera and a NIS Elements Advanced (version 4.30) program was used to visualize and record migration data in 15 minute intervals over a period of 18 hours. ASCs seeded on three stiffness gradient $(n=3)$ and three static $(n=3)$ GelMA hydrogels were placed in an OKOLab chamber where temperature, $\mathrm{CO}_{2}$ and humidity was controlled: $37^{\circ} \mathrm{C} / 5 \% \mathrm{CO}_{2}$. Cells were manually tracked using Fiji ImageJ software and migration data was analyzed via GraphPad Prism to determine individual cell paths and $X, Y$ velocities. [9]

\subsection{Immunofluorescence}

After designated culture periods, normal hydrogels and inhibitor-treated hydrogels were washed with PBS and fixed with 4\% paraformaldehyde (PFA; ChemCruz) for 15 minutes at room temperature. Following PBS washes (three washes for 2 minutes each), ASCs were then permeabilized using 0.2\% Triton-X 100 (Sigma) for 30 minutes at room temperature. Fixed and permeabilized hydrogels were transferred to polydimethylsiloxane (PDMS)-coated 6-well plates and incubated for one hour at $37^{\circ} \mathrm{C} / 5 \% \mathrm{CO}_{2}$ with $5 \%$ goat serum to block nonspecific antibody binding. All the following primary antibodies were purchased from Santa Cruz unless otherwise stated, and used in $2 \%$ bovine serum albumin (BSA; Sigma). Post PBS washes, ASCs were incubated at $37^{\circ} \mathrm{C}$ for one hour covered in $200 \mu \mathrm{L}$ of primary antibody solutions at 
1 YAP (sc-101199), MRTF-A (sc-390324), PPAR $\quad(\mathrm{C} 26 \mathrm{H} 12 ; \quad$ Cell Signaling 2 Technologies) or MyoD (MABE132; MERCK). Blebbistatin and Y-27632 treated samples were stained only for LaminA and YAP. Primary staining was followed by PBS washes and secondary antibodies of either goat anti-mouse Alexa Fluor 594 (ab150116; Abcam), goat anti-rabbit Alexa Fluor 594 (ab150080; Abcam) or goat antimouse Alexa Fluor 647 (ab150115; Abcam). Hydrogels covered in $200 \mu \mathrm{L}$ solutions (1:200) of corresponding secondary antibodies and Alexa Fluor 488 Phalloidin (Gibco), were incubated at $37^{\circ} \mathrm{C}$ for another hour then washed. Samples were then incubated with DAPI at 1:500 in PBS for 10 minutes at room temperature then washed. Coverslips were mounted onto slides with Fluoroshield mounting solution (Sigma) and sealed with clear nail polish. A Nikon $\mathrm{C} 2+$ confocal microscope using NIS Elements Advanced (4.0) software (Nikon) was used to image samples. [9]

\subsection{Data collection and statistical analyses}

Images for mechanosensor and differentiation marker expression were obtained across the $15 \mathrm{~mm}$ stiffness gradient in triplicates for three samples per marker $(n=3)$. Images were analyzed using a custom pipeline designed in CellProfiler. All data sets are expressed as mean \pm standard error of the mean of experiments unless otherwise stated. Linear regression $R$ squared values were obtained for mechanosensor expression trends and statistical comparison of $X$ and $Y$ velocities were done via an unpaired student's $t$-test $(P<0.05$ considered statistically significant). For fitting, either exponential or linear fits were used based on the presence or absence, respectively, of regions of stiffness-insensitive expression patterns (i.e. saturation regions).

\section{Results and Discussion}

\subsection{Fabrication of a linear stiffness gradient hydrogel}

The fabrication of a stiffness gradient GelMA hydrogel is schematically shown in Figure 1A. Utilising a glass polymerisation surface, which involves a sandwich of GelMA between a coverslip and a microscope slide, 10\% GeIMA was polymerised for 90 seconds through a photomask of $20-100 \%$ transparency. Photomasks, designed on PowerPoint, were printed onto transparent films as $15 \times 15 \mathrm{~mm}$ squares. The compressive Young's Moduli of the gradient hydrogel was measured by atomic force 
1 microscopy (AFM). Triplicate indentations taken across the surface of the gel 2 generated contact force graphs which were analysed to produce the stiffness (in $\mathrm{kPa}$ ) 3 over length of the hydrogel (in $\mathrm{mm}$ ) graphs shown in Figure 1B. Linear regression 4 analysis revealed a stiffness gradient of $0.68 \mathrm{kPa} / \mathrm{mm}\left(\mathrm{R}^{2}=0.9697\right)$ ranging from 3.5 $5 \pm 0.3$ to $13.1 \pm 0.7 \mathrm{kPa}$. This stiffness range covers the physiological stiffness range 6 of adipogenic $(\sim 4 \mathrm{kPa})$ to myogenic tissues $(\sim 12 \mathrm{kPa})$, which adipose-derived stem 7 cells (ASCs) have been shown to differentiate in a specific tissue stiffness-dependent 8 manner. [3, 9] We thus chose this regime in order to investigate ASC responses on our GelMA gradient system. We also showed that by changing the photomask to 10 - $100 \%$ transparency, the gradient strength could be increased to $0.96 \mathrm{kPa} / \mathrm{mm}$, ranging from $5.4 \pm 0.3$ to $18.5 \pm 0.7 \mathrm{kPa}$ over the length of the hydrogel (Figure S1,

12 Supporting Information).

A

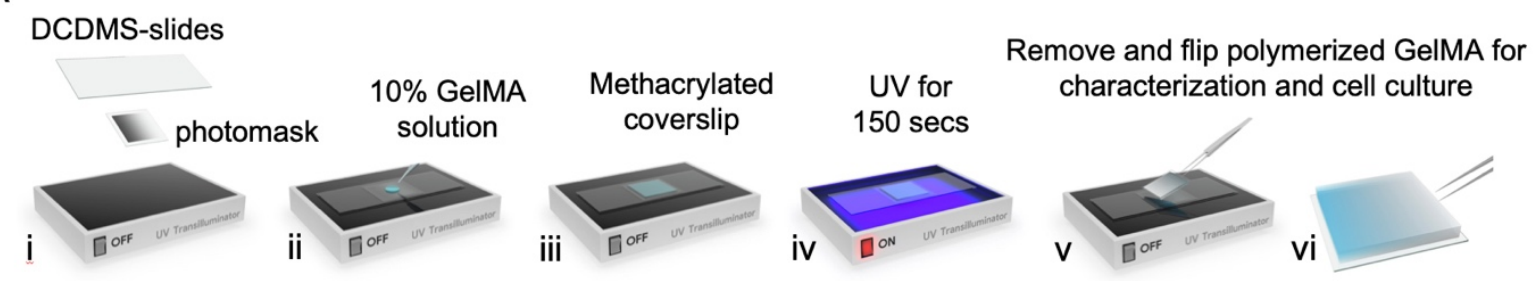

B

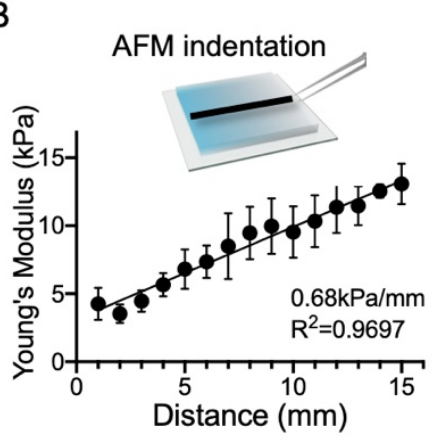

C
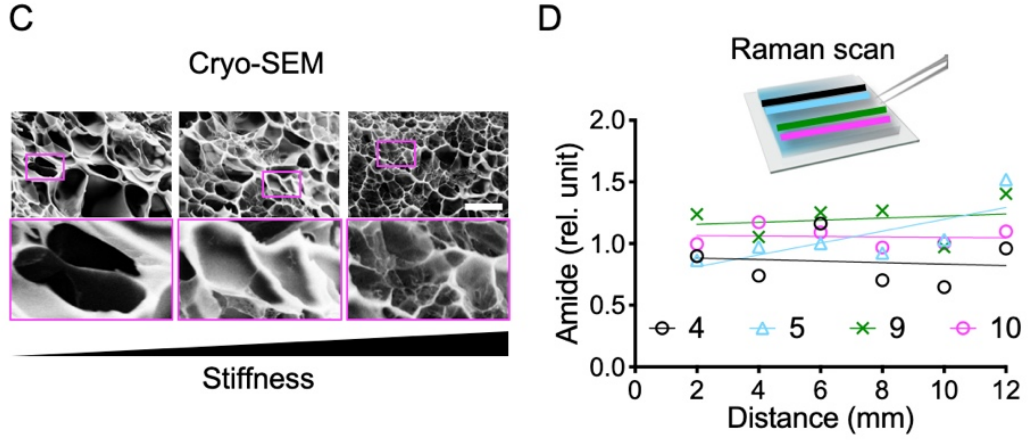

Figure 1. GelMA stiffness gradient hydrogel fabrication and characterization. A. Schematic of UV polymerization of stiffness gradient GelMA hydrogel using a photomask. B. AFM measurement on GelMA stiffness gradient hydrogel $(n=4)$. C. Pore size investigation with images from cryo-SEM showed an inverse correlation to stiffness. Scale bar is $5 \mu \mathrm{m}$. Insets show zoomed in regions of interest.

D. Raman showed no significant changes in amide amount across the stiffness gradient. Raman measurements were done across the stiffness gradient over 5 points in 4 different lines at 4,5,9 and $10 \mathrm{~mm}$ from the top of the gel. Data are shown as mean \pm SEM.

23 Polymer networks and topography across the surface of the gradient hydrogel were visualized via scanning electron microscopy (SEM). Figure $1 \mathrm{C}$ depicts the inverse 
1 relationship between pore size and stiffness along the gradient, indicating that GeIMA 2 networks become denser with increasing stiffness. Raman spectroscopy showed no 3 changes in amide amount parallel or perpendicular to the gradient (Figure 1D and S1B, 4 Supporting Information). The data suggests that there was an increase in crosslinking

5 (GelMA polymer network not GelMA concentration) with increasing stiffness.

6

\section{$7 \quad 3.2$ Non-durotactic gradient}

8 Previous research regarding cell migration has shown that cells preferentially migrate 9 from soft to stiffer regions - a process known as durotaxis. [8, 9, 12-14] In hydrogel systems with gradients strengths greater than $8.2 \mathrm{kPa} / \mathrm{mm}$, cells undergo active durotaxis. [9] In our system, in which we aim to characterise mechanosensitive protein expression across the gradient, this biased movement would confound protein expression results, hence our aim was to develop a gradient that did not induce durotactic behaviour. In Figure S2, we show results of cell migration tracked over 18 hours on discrete, i.e. no stiffness gradient, gels vs. stiffness gradient gels. Migration paths of individual cells on both gels show no favoured direction of movement and no significant difference was observed between $X$ and $Y$ velocities from the overall population. This leads us to conclude that our stiffness gradient of $0.68 \mathrm{kPa} / \mathrm{mm}$ did not induce durotaxis as would be expected. Therefore, ASCs were evenly distributed, and functional outputs could be directly attributed to mechanosensation at the local substrate stiffness (Figure 2A). 
A

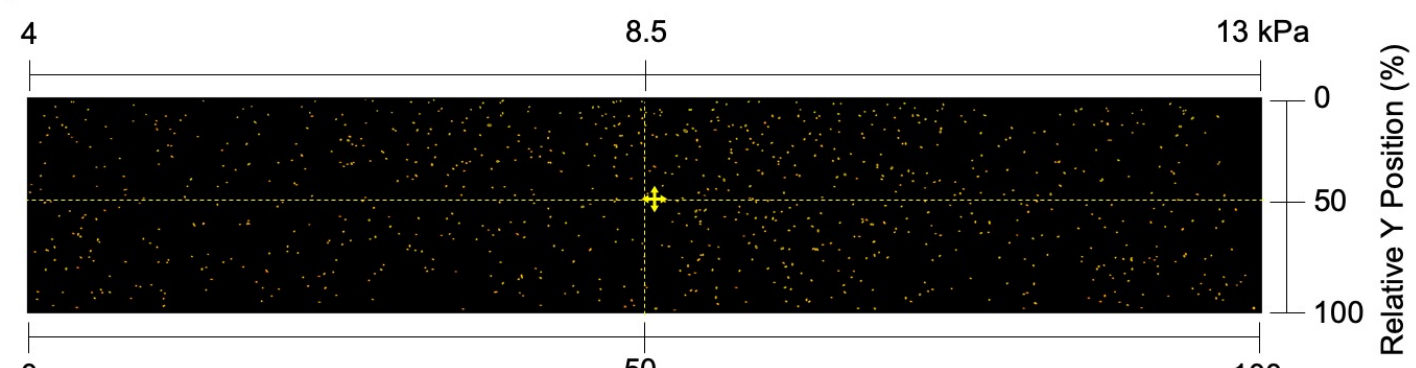

0

50

100

Relative X Position (\%)
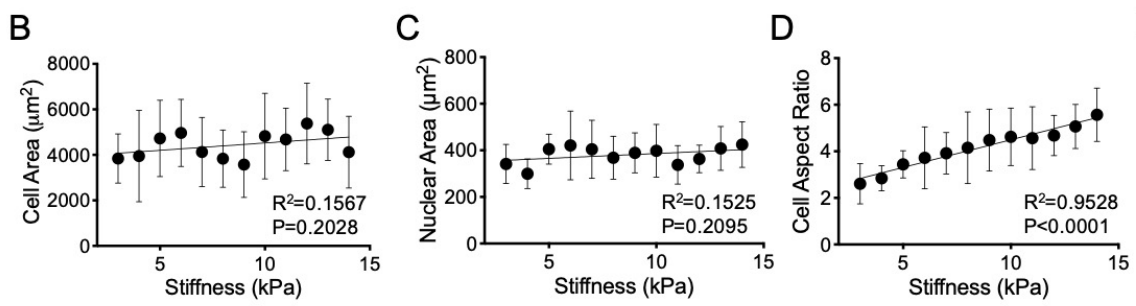

E

$\mathrm{F}$
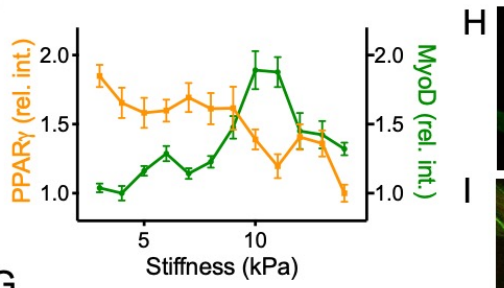

G
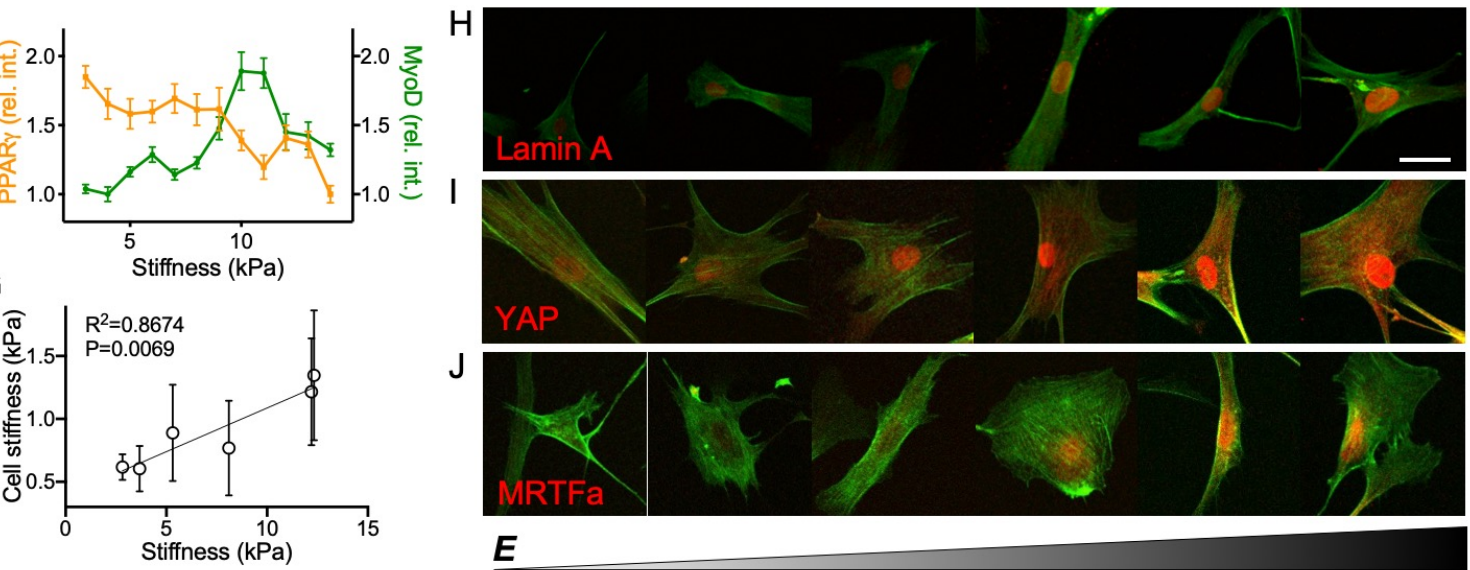

$\mathrm{J}$

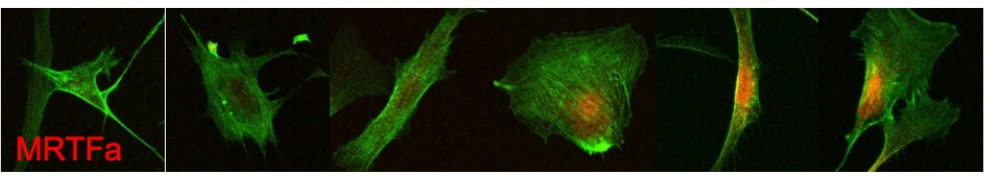

$\boldsymbol{E}$

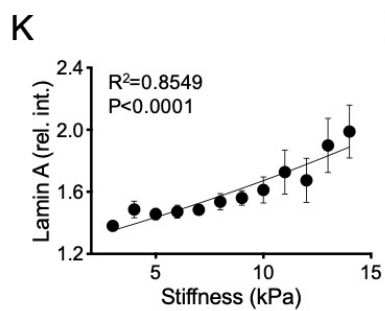

L

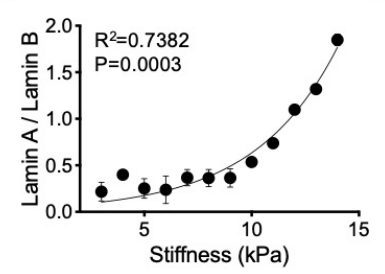

M

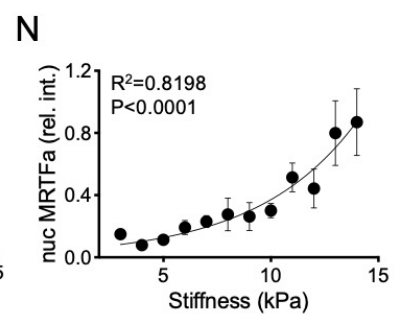

Figure 2. Morphological characteristics, mechanosensation, and differentiation of ASCs on stiffness gradient GelMA hydrogel. A. Even distribution of cells was visualised by Dapi staining and a centroid of cells (yellow cross). B-E. Morphological parameters and K-N. mechanomarkers are plotted as a function of hydrogel stiffness. H-J. Representative images for Lamin A, YAP and MRTFa staining. Scale bar is $20 \mu \mathrm{m}$. G. Cell stiffness measured by AFM displayed per hydrogel stiffness. F. Relative intensity of PPARY (orange) and MyoD (green) are presented as a function of hydrogel stiffness. ASCs were cultured for 3 days except differentiation experiment which was 5 days $(F)$. Data are shown as mean $\pm S E M . R^{2}$ and $P$ values are from correlation analyses with either linear or exponential fits. 


\section{$1 \quad 3.3$ Stem cell morphology and differentiation are related to stiffness}

2 Cells can alter their size and shape based on their environment; thus, differences in observed cell morphology are indicative of differential mechanosensation. [9] No trend in ASC cell area or nuclear area was observed along the stiffness gradient (Figure 2B, $C$ and $E$ ), however, an increase in cell aspect ratio with increasing stiffness was observed (Figure 2D). Unlike previous observations using polyacrylamide gradient hydrogels, [9] ASCs showed no clear correlation between nuclear and cell area on the

8 GelMA stiffness gradient hydrogels (Figure S3, Supporting Information). This can be explained by the morphologies seen in corresponding physiological tissues in vivo. Adipogenic tissues have more rounded cell shapes compared to myogenic tissues which are more elongated and spindle-shaped. Aspect ratios calculated from height (Y length) over base (X length) of a cell would therefore exhibit larger ratios in spindleshaped cells compared to more circular cells. Hence, in the lower stiffness range of our gels, $3.5 \mathrm{kPa}$, which induces adipogenic differentiation, we measured aspect ratios between 2 and 3, whereas in the myogenic stiffness region of our gels, $\sim 12 \mathrm{kPa}$, which induces myogenic differentiation, we observe larger aspect ratios between 5 and 6 (Figure 2D). The influence of stiffness on lineage-specific differentiation related to the morphological measurements was examined by analysing the expression of early adipogenic and myogenic markers of differentiation. To do this, we stained for both PPAR $\gamma$, an adipogenic marker, and MyoD, a myogenic marker along our stiffness gradients and found PPAR peak expression in softer regimes and MyoD peak expression in stiffer regimes as expected when normalized relative intensity compared over stiffness ranges (Figure 2F). The upregulation of PPAR $\gamma$ at low stiffnesses and its gradual reduction as stiffness increases to $\sim 15 \mathrm{kPa}$ is in contrast with the sharp stiffness-sensitive expression of MyoD (Figure 2F), suggesting that the 'width' of stiffness-sensitive differentiation may play an important tissue-specific role in homeostasis (i.e. adipogenesis can occur in a wider variety of stiffnesses, while myogenesis requires a highly regulated mechanical microenvironment).

\subsection{Stem cells mechanosense across a spectrum of ECM stiffness}

To determine stiffness-dependent effects of mechanosensation in ASCs on our hydrogels, we measured expression of well-known mechanosensitive proteins Lamin A, a nuclear intermediate filament; Yes-associated protein (YAP), a nuclear co- 
1 transcriptional factor; and Myocardin-related transcription factor A (MRTFa, also

2 known as MAL), a transcriptional co-activator of serum response factor. [9-11, 23] In

3 physiological tissues, it has been shown that Lamin A expression scales with 4 increasing tissue stiffness; this has also been shown to be true in vitro on hydrogel 5 systems. [11] YAP is a mechanosensitive protein that functions as a nuclear relay 6 between the nucleus and the cytoplasm, alternating its localisation depending on the 7 mechanical signal. ${ }^{[10]}$ In stiff environments, YAP localizes within the nucleus and on 8 soft, it remains within the cytoplasm. Although the exact mechanism of 9 mechanotransduction is unclear, YAP has been shown to be crucial in stem cell differentiation induced by stiffness and can override mechanical cues when overexpressed. [24] Lastly, the activity of MRTFa is directly related to the actin polymerisation level of the cell.[25] As a protein that shuttles between the nucleus and cytoplasm in response to mechanical signals, its activity is closely related to that of YAP. Recent studies have also shown it plays a role in transcriptional regulation and cell proliferation. ${ }^{[26]}$

Figure 2H-J show representative images for Lamin A, YAP, and MRTFa immunocytochemistry and graphs in Figure $2 \mathrm{~K}-\mathrm{N}$ show the quantified fluorescence intensity of each marker along the stiffness gradient. All three mechanosensors exhibit an increase in expression with increasing stiffness. Furthermore, we measured the stiffness of ASCs by AFM and found that it was highly correlated to increasing substrate stiffness (Figure 2G).

\subsection{Disrupting cellular contractility prevents mechanotransduction}

We next wanted to understand the role of the actin cytoskeleton in transducing mechanical signals. Thus, we treated ASCs with two actomyosin-disrupting agents, Blebbistatin and Y-27632. As a myosin II inhibitor, Blebbistatin prevents proper actomyosin cross-linking within the cytoskeleton of cells thereby disrupting the cell's ability to maintain shape, move and proliferate. [27] Y-27632 is a Rho-associated protein kinase (ROCK) inhibitor. The ROCK family is an important regulator of actomyosin contractility; therefore, Y-27632 interferes with cell adhesion, division, shape, and movement. [28] When introduced into culture, Blebbistatin and Y-27632 completely abolish mechanotransduction, as demonstrated by no correlation in all measured outputs (except nuclear aspect ratio) vs. stiffness (Figure 3 and S4-6, 
1 Supporting Information). As these inhibitors disrupt the actin cytoskeleton, we observe

2 alterations in normal ASC morphologies, indicated by a decrease in both cell and 3 nuclear size.

4
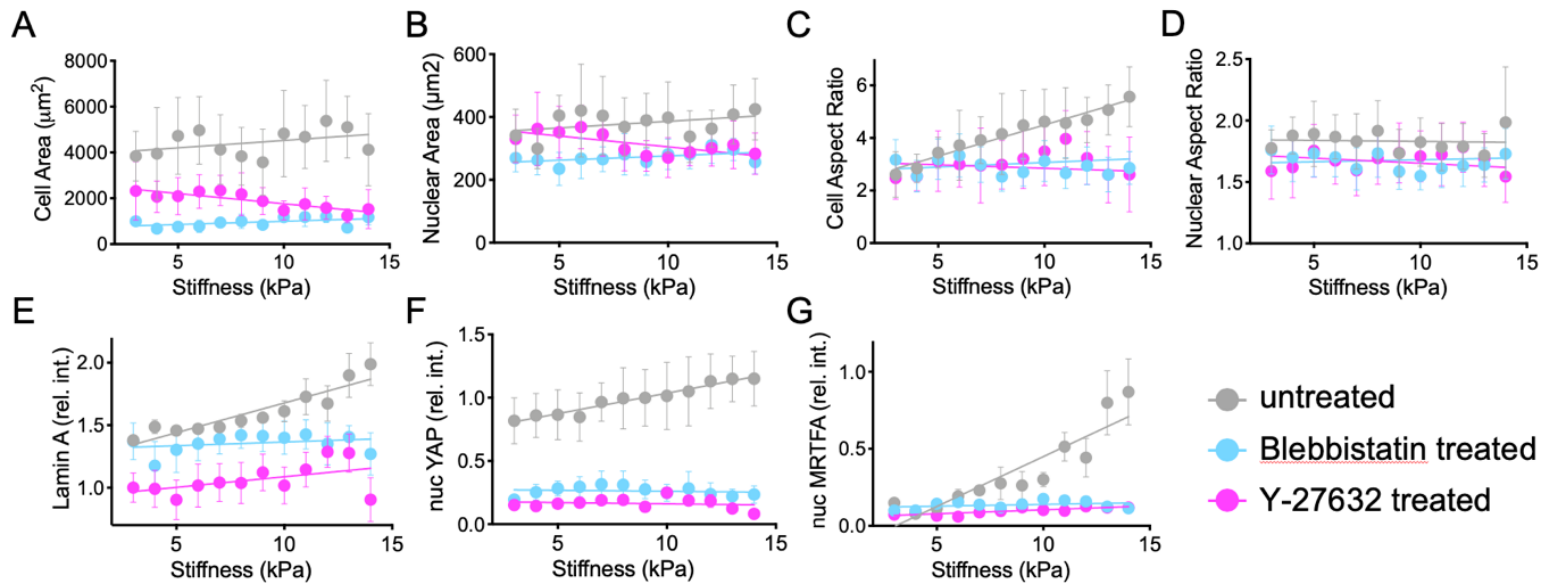

Figure 3. The effect of inhibition in morphological characteristics, mechanosensation, and differentiation of ASCs on stiffness gradient GeIMA hydrogel. A-D. Morphological parameters and E-G. Mechanomarkers are plotted as a function of hydrogel stiffness. Grey: untreated, Sky blue: Blebbistatin treated, and Magenta: Y-27632 treated. Data are shown as mean \pm SEM. Linear regression was used to fit data.

\section{Conclusions}

Stiffness gradient GelMA hydrogels provide a platform to screen stem cell mechanotransduction, differentiation, and migration over a wide range of physiological tissue stiffness values in one sample. In this study, shallow gradient hydrogels fabricated via UV polymerization through a gradient photomask enable screening of both phenotypic and genotypic changes in ASCs at high-resolution. While this study employed only one gradient strength $(0.68 \mathrm{kPa} / \mathrm{mm})$ due to the fact that it did not induce durotaxis, variations of this platform with simple modification to the gradient photomask or hydrogel components can enable a diverse range of stiffness gradients for various applications in cell biology. The nature of GelMA also affords for the possibility of generating a gradient in three dimensions after cell encapsulation, thereby making it a highly versatile platform for cellular studies. Indeed, work from our group has shown that in stem cells encapsulated within 3D GelMA stiffness gradient hydrogels, an inverse stiffness-sensitive relationship is observed compared to patterns found on 2D gradients. [29] This observation is also supported by recent investigations 
1 into the interplay between cell volume, mechanotransduction, and stem cell 2 differentiation. [30, 31] Intriguingly, our GelMA gradient tool is compatible with 3 simultaneous 2D and 3D gradient stiffness investigations in a single well. Future work 4 will undoubtedly continue to decouple the respective contributions of cell spreading 5 and expansion, matrix-induced confinement, and mechanical properties of the 6 microenvironment.

\section{Acknowledgements}

9 This study work was supported by National Health and Medical Research Council Grant PG1098449 (to YS.C), Heart Foundation Future Leader Fellowship 101173 (to YS.C), Department of Health, Western Australia, Merit awards - project and fellowship (to YS.C), and Universities Australia DAAD German Research Cooperation 5744610 (to YS.C, C.K, A.W.H, J.L.Y and J.P.S).

\section{Author contributions}

C.K. L.G.M. and YS.C designed and planned the study. J.L.Y., A.W.H., and J.P.S. performed SEM and pore size analysis. K.J. and Z.M.A. performed Raman spectroscopy. JH.J., Y.H. and D-W.H synthesized GelMA. C.K., J.L.Y. and YS.C drafted the manuscript. YS.C provided supervision and funding. All authors discussed the data and contributed to the final version of the manuscript.

\section{References}

[1] A.J. Engler, S. Sen, H.L. Sweeney, D.E. Discher, Matrix elasticity directs stem cell lineage specification, Cell 126(4) (2006) 677-89.

[2] G.C. Reilly, A.J. Engler, Intrinsic extracellular matrix properties regulate stem cell differentiation, Journal of biomechanics 43(1) (2010) 55-62.

[3] Y.S. Choi, L.G. Vincent, A.R. Lee, M.K. Dobke, A.J. Engler, Mechanical derivation of functional myotubes from adipose-derived stem cells, Biomaterials 33(8) (2012) 2482-91.

[4] D.E. Discher, P. Janmey, Y.L. Wang, Tissue cells feel and respond to the stiffness of their substrate, Science (New York, N.Y.) 310(5751) (2005) 1139-43. 
1 [5] C.L. Happe, K.P. Tenerelli, A.K. Gromova, F. Kolb, A.J. Engler, Mechanically patterned

2 neuromuscular junctions-in-a-dish have improved functional maturation, Molecular biology of

3 the cell 28(14) (2017) 1950-1958.

4 [6] M.F. Berry, A.J. Engler, Y.J. Woo, T.J. Pirolli, L.T. Bish, V. Jayasankar, K.J. Morine, T.J.

5 Gardner, D.E. Discher, H.L. Sweeney, Mesenchymal stem cell injection after myocardial

6 infarction improves myocardial compliance, American journal of physiology. Heart and

7 circulatory physiology 290(6) (2006) H2196-203.

8 [7] L.M. Cross, K. Shah, S. Palani, C.W. Peak, A.K. Gaharwar, Gradient nanocomposite 9 hydrogels for interface tissue engineering, Nanomedicine : nanotechnology, biology, and 10 medicine 14(7) (2018) 2465-2474.

11 [8] J.R. Tse, A.J. Engler, Stiffness gradients mimicking in vivo tissue variation regulate mesenchymal stem cell fate, PloS one 6(1) (2011) e15978.

[9] W.J. Hadden, J.L. Young, A.W. Holle, M.L. McFetridge, D.Y. Kim, P. Wijesinghe, H.

Taylor-Weiner, J.H. Wen, A.R. Lee, K. Bieback, B.N. Vo, D.D. Sampson, B.F. Kennedy, J.P.

Spatz, A.J. Engler, Y.S. Choi, Stem cell migration and mechanotransduction on linear stiffness

gradient hydrogels, Proceedings of the National Academy of Sciences of the United States of America 114(22) (2017) 5647-5652.

[10] S. Dupont, L. Morsut, M. Aragona, E. Enzo, S. Giulitti, M. Cordenonsi, F. Zanconato, J.

Le Digabel, M. Forcato, S. Bicciato, N. Elvassore, S. Piccolo, Role of YAP/TAZ in mechanotransduction, Nature 474(7350) (2011) 179-83.

21 [11] J. Swift, I.L. Ivanovska, A. Buxboim, T. Harada, P.C. Dingal, J. Pinter, J.D. Pajerowski, K.R. Spinler, J.W. Shin, M. Tewari, F. Rehfeldt, D.W. Speicher, D.E. Discher, Nuclear laminA scales with tissue stiffness and enhances matrix-directed differentiation, Science (New York, N.Y.) 341(6149) (2013) 1240104. 
1 [12] Y.S. Choi, L.G. Vincent, A.R. Lee, K.C. Kretchmer, S. Chirasatitsin, M.K. Dobke, A.J.

2 Engler, The alignment and fusion assembly of adipose-derived stem cells on mechanically 3 patterned matrices, Biomaterials 33(29) (2012) 6943-51.

4 [13] C.D. Hartman, B.C. Isenberg, S.G. Chua, J.Y. Wong, Vascular smooth muscle cell 5 durotaxis depends on extracellular matrix composition, Proceedings of the National Academy 6 of Sciences of the United States of America 113(40) (2016) 11190-11195.

7 [14] L.G. Vincent, Y.S. Choi, B. Alonso-Latorre, J.C. del Alamo, A.J. Engler, Mesenchymal 8 stem cell durotaxis depends on substrate stiffness gradient strength, Biotechnology journal 8(4) 9 (2013) 472-84.

10 [15] J. Leijten, J. Seo, K. Yue, G.T. Santiago, A. Tamayol, G.U. Ruiz-Esparza, S.R. Shin, R. 11 Sharifi, I. Noshadi, M.M. Alvarez, Y.S. Zhang, A. Khademhosseini, Spatially and Temporally 12 Controlled Hydrogels for Tissue Engineering, Materials science \& engineering. R, Reports : a review journal 119 (2017) 1-35.

[16] A.M. Kloxin, J.A. Benton, K.S. Anseth, In situ elasticity modulation with dynamic substrates to direct cell phenotype, Biomaterials 31(1) (2010) 1-8. [17] C.D. O'Connell, B. Zhang, C. Onofrillo, S. Duchi, R. Blanchard, A. Quigley, J. Bourke, 17 S. Gambhir, R. Kapsa, C. Di Bella, P. Choong, G.G. Wallace, Tailoring the mechanical properties of gelatin methacryloyl hydrogels through manipulation of the photocrosslinking conditions, Soft matter 14(11) (2018) 2142-2151.

[18] F. Piraino, G. Camci-Unal, M.J. Hancock, M. Rasponi, A. Khademhosseini, Multigradient hydrogels produced layer by layer with capillary flow and crosslinking in open microchannels, Lab on a chip 12(3) (2012) 659-61. methacrylate-based matrices induce osteogenic differentiation of human induced pluripotent stem cells, Acta biomaterialia 10(12) (2014) 4961-4970. 
1 [20] N. Colthup, L. Daly, S. Wiberley, Introduction to Infrared and Raman Spectroscopy (Third

2 Edition). Introduction to Infrared and Raman Spectroscopy (Third Edition), 1990.

3 [21] P. Stiles, J. Dieringer, N. Shah, Surface-Enhanced Raman Spectroscopy, Annual Review

4 of Analytical Chemistry 1 (2008) 601-26.

5 [22] M. Janko, A. Zink, A. Gigler, W. Heckl, R. Stark, Nanostructure and mechanics of

6 mummified type I collagen from the 5300-year-old tyrolean Iceman, Proceedings of the Royal

7 Society B: Biological Sciences 277(1692) (2010).

8 [23] O.M. Yu, S. Miyamoto, J.H. Brown, Myocardin-Related Transcription Factor A and Yes-

9 Associated Protein Exert Dual Control in G Protein-Coupled Receptor- and RhoA-Mediated

10 Transcriptional Regulation and Cell Proliferation, Molecular and cellular biology 36(1) (2016) $11 \quad 39-49$.

[24] G. Nardone, J. Oliver-De La Cruz, J. Vrbsky, C. Martini, J. Pribyl, P. Skladal, M. Pesl, G.

Caluori, S. Pagliari, F. Martino, Z. Maceckova, M. Hajduch, A. Sanz-Garcia, N.M. Pugno,

G.B. Stokin, G. Forte, YAP regulates cell mechanics by controlling focal adhesion assembly,

$15 \quad$ Nature communications 8 (2017) 15321.

16 [25] F. Miralles, G. Posern, A.I. Zaromytidou, R. Treisman, Actin dynamics control SRF 17 activity by regulation of its coactivator MAL, Cell 113(3) (2003) 329-42. [26] O.M. Yu, S. Miyamoto, J.H. Brown, Myocardin-Related Transcription Factor A and YesAssociated Protein Exert Dual Control in G Protein-Coupled Receptor- and RhoA-Mediated Transcriptional Regulation and Cell Proliferation, Molecular and cellular biology 36(1) (2015) $39-49$.

[27] M. Kovacs, J. Toth, C. Hetenyi, A. Malnasi-Csizmadia, J.R. Sellers, Mechanism of blebbistatin inhibition of myosin II, The Journal of biological chemistry 279(34) (2004) 3555763. 
1 [28] S. Kumper, C.J. Marshall, ROCK-driven actomyosin contractility induces tissue stiffness

2 and tumor growth, Cancer cell 19(6) (2011) 695-7.

3 [29] L.G. Major, A.W. Holle, J.L. Young, M.S. Hepburn, K. Jeong, I.L. Chin, R.W. Sanderson,

4 J.H. Jeong, Z.M. Aman, B.F. Kennedy, Y. Hwang, D.W. Han, H.W. Park, K.L. Guan, J.P.

5 Spatz, Y.S. Choi, Volume adaptation controls stem cell mechanotransduction, ACS applied

6 materials \& interfaces (2019).

7 [30] S.R. Caliari, S.L. Vega, M. Kwon, E.M. Soulas, J.A. Burdick, Dimensionality and

8 spreading influence MSC YAP/TAZ signaling in hydrogel environments, Biomaterials 103

9 (2016) 314-323.

10 [31] H.P. Lee, R. Stowers, O. Chaudhuri, Volume expansion and TRPV4 activation regulate

11 stem cell fate in three-dimensional microenvironments, Nature communications 10(1) (2019)

12529.

13 